\title{
Aportes a la valoración ambiental desde el análisis de beneficios y costos de establecer un parque en la margen izquierda del río Sinú, Montería, Colombia ${ }^{1}$
}

\section{Contribution to environmental assessment based on analysis benefits and costs to establish a park on the left-hand bank of the Sinú river, Montería, Colombia}

\section{Rubén Darío Sepúlveda Vargas²}

\section{Resumen}

El presente estudio intenta valorar económicamente los beneficios y los costos de crear un parque en la margen izquierda del río Sinú en la Ciudad de Montería, Colombia, con el fin de facilitar las decisiones de políticas públicas ambientales en el marco de la gestión del territorio. La medición de los beneficios se modela por el método y valoración contingente, mientras que la estimación de los costos por el método costo de oportunidad. Los resultados indican que la decisión de crear el parque es viable económicamente al arrojar una relación beneficios-costos $(B / C)$ de 1,47 incluyendo en el análisis sólo las viviendas de estratos 1 y 2, y una relación $B / C$ de 2,31 con todas las viviendas de estratos socioeconómicos.

Palabras clave: Economía del bienestar; Externalidades; Valoración contingente; Costo de oportunidad; Análisis costos-beneficios.

\begin{abstract}
His study attempts to assess economic benefits and costs of creating a park on the left bank of the Sinu river in the city of Monteria, Colombia, in order to facilitate environmental policy decisions in the context of land management. The measurement of benefits is modeled by the contingent valuation method and, whiles the cost estimate by the opportunity cost method. The results indicate that the decision to create the park is economically feasible to throw a benefit-cost ratio $(B / C)$ of 1.47 in the analysis including only those households in strata 1 and 2 , and a ratio $B / C$ of 2.31 in all socioeconomic strata housing.
\end{abstract}

Keywords: Welfare economics; Externalities; Contingent valuation; Cost-benefit analysis.

\section{Introducción}

Montería, capital del departamento de Córdoba, esta ubicada a orillas del río Sinú, con una población de 381.525 habitantes (según el censo de población de 2005), es uno de los centros económicos y culturales más importantes de la costa Caribe colombiana. Presenta un gran avance en su desarrollo arquitectónico, comercial y vial. Simultáneamente se han creado varios parques en toda la ciudad, siendo el de mayor relevancia el que se encuentra ubicado en el margen
1 Este artículo es producto del proyecto de investigación que lleva su mismo nombre, financiado por la Universidad Pontificia Bolivariana seccional Montería. Este proyecto se inició en agosto de 2008 y se finalizó en junio de 2010. El proyecto se enmarca dentro de la sub-línea de investigación de «economía ambiental» de la Facultad de Economía. En la realización participó el docente Jairo Ángel Guzmán y los estudiantes miembros del semillero Jaime Rangel Bolaños, Margarita Miranda y Juan Alejandro Galeano. Se agradece al Ingeniero Carlos Montoya de la Secretaría de Infraestructura de la Alcaldía de Montería por los datos suministrados.

2 Economista, Universidad Jorge Tadeo Lozano, Bogotá, Colombia. Magíster en Economía, Pontificia Universidad Javeriana, Bogotá, Colombia. Profesor Titular en la Facultad de Economía de la Universidad Pontificia Bolivariana seccional de Montería, Colombia. Docente Titular Facultad de Economía. Creador y coordinador del semillero de economía ambiental «SEA», Montería, Colombia.

e-mail: ruben.sepúlveda@upbmonteria. edu.co

Recibido: 1 de febrero de 2012

Aceptado: 7 de marzo de 2012 


\section{Bioetnia Volumen 9 No 1 (enero-junio), 2012}

futuro inmediato. Los moradores han dependido tradicionalmente de actividades comerciales de menor escala, entre ellas tiendas, restaurantes, venta de minutos a celular, venta de gaseosas y cervezas, venta de carne de res, remontadora de calzado, entre otras. Las características de los negocios son pequeñas unidades de comercio que se encuentran ubicadas en el mismo lugar de residencia, es decir habilitan un cuarto o parte de la casa para desarrollar la actividad comercial. Los ingresos que se generan por estas actividades son relativamente bajos, los hacen para generar un ingreso complementario o por situación extrema de desempleado.

En esta investigación se pudo constatar según los encuestados, que el mayor costo se asocia con la pérdida de propiedad más que a renunciar a las actividades comerciales. Es por ello que en la metodología de valoración del costo de oportunidad, el concepto que se tuvo en cuenta fue la vivienda. Además de los costos asociados con la pérdida de la vivienda y de actividades comerciales, se encuentran los costos de la erosión por deslizamiento del terreno -información que no se conoce y no fue posible conseguirla en la Secretaría de Infraestructura Municipal- y los costos de construcción del parque, que existe información y se utilizó en los cálculos del proyecto para determinar la relación beneficio/costo $\mathrm{B} / \mathrm{C}$.

La segunda parte del estudio se enfocó a estimar los beneficios del establecimiento del parque. El método de valoración contingente permitió medir la DAP de una muestra de 223 casas ubicadas muy cerca del lugar donde se crearía el parque. Luego de haber determinado el valor de DAP se extrapoló a toda las casa del municipio con el fin de calcular el valor promedio de ingresos. En la investigación se tuvo en cuenta que existen beneficiarios directos e indirectos. Los primeros aquellos que están afectados de forma positiva y directa por la construcción del parque, por ejemplo, los que residen al frente o entre una o cuatro calles de distancia. Y los segundos los que están afectados de forma positiva e indirecta, por ejemplo, los que residen en la margen derecha del río Sinú.

El artículo se divide en cuatro partes. En la primera se describe la teoría y conceptos de la valoración económica del medio ambiente, en especial valoración contingente y costo de oportunidad. En la segunda se detalla la metodología y la determinación de la muestra seleccionada. En la tercera parte se analiza los resultados desde el punto de vista de la estadística descriptiva y modelo econométrico utilizado. Finalmente, en la última parte se presenta las principales conclusiones del trabajo.

\section{Teoría de la valoración económica del medio ambiente}

El método de valoración contingente. Es común encon- trar casos en que no existe un mercado para bienes y servicios ambientales, no están bien definidos o no hay mercados alternativos, en consecuencia, es difícil valorar los efectos ambientales de un proyecto que se pretenda realizar o se haya realizado. No obstante existe una alternativa, anclada en la teoría económica de la demanda del consumidor, comúnmente llamada método de valoración contingente (MVC), que pretende realizar una valoración económica hipotética de aquellos bienes y servicios ambientales ausentes de mercados. Esta valoración se realiza a través de técnicas de encuestas en la que se le pregunta al usuario del activo ambiental su disposición a pagar por una eventual mejora.

Este es el caso de un parque, con características de bien público y por tanto es necesario determinar el valor que tiene el parque para la comunidad, con fines de protección, conservación o mejoramiento. Si los encuestados expresan disposición a pago, entonces el parque le genera beneficios en términos de utilidad o satisfacción. Normalmente los parques incorporan una serie de funciones ambientales y económicas que la comunidad desea usar para disfrute presente y futuro. Sepúlveda (2008) presenta las funciones económicas y ambientales del parque Ronda del Sinú de la ciudad de Montería. Estas las clasifica en valor de uso directo e indirecto y valor de no uso, entre ellas transporte acuático, abastecimiento de agua, recreación y deporte, estabilización de microclima, biodiversidad, patrimonio cultural, pesca, transporte terrestre, comercio y salón de conferencia.

De acuerdo con Mathews et al. (1995), la metodología de valoración contingente es un método utilizado ampliamente para bienes y servicios que no se transan en el mercado, pertenece a la familia o conjunto de métodos llamados preferencia revelada. El supuesto fundamental del método es que los individuos muestran el mismo comportamiento en un mercado hipotético (o contingente) frente a un mercado real.

Riera (1994) trata de averiguar la disposición a pagar del encuestado por disfrutar un bien ambiental. Esta forma de medición le permite una amplitud de aplicaciones en la valoración económica de bienes ambientales, pasando por determinación de daños de bienes colectivos hasta por mejoras de bienes públicos.

Azqueta (1994) indica que la metodología para obtener información por medio de la pregunta directa es posible hacerla de varias formas como entrevistas personales o telefónicas, encuestas por correo, experimentos en laboratorios. En todas estos abordajes al entrevistado, se realiza la pregunta sobre su disposición a pagar bajo diferentes formatos, es decir, que hay cierta flexibilidad para mejorar la captura de información por medio de la forma de preguntar. Entre los formatos hasta ahora desarrollados se encuentran, abierto, subasta, múltiple, binario e iterativo.

El MVC normalmente utiliza los modelos de estimación de Logit y/o Probit, que permite estudiar la probabilidad de 
que una persona o grupo de personas al momento de ser encuestada responda si está de acuerdo o no a pagar por un cambio o mejora en las condiciones ambientales. Es decir, el MVC emplea un modelo de elección cualitativa que analiza la probabilidad de que un individuo con un conjunto dado de atributos como edad, sexo, ingresos, educación, entre otros, efectúe una elección determinada. Usualmente el modelo se plantea de la siguiente manera:

$$
P(\mathrm{Y}=1)=\frac{1}{1+\mathrm{e}^{(\alpha+\beta \times \mathrm{i})}}
$$

A través de este modelo tratamos de estimar la probabilidad de que la variable dependiente tenga un valor igual a uno, es decir en nuestro caso la probabilidad de que un individuo esté dispuesto a pagar el precio hipotético de disfrutar el parque que se creará, en este caso es la variable dependiente. Y ello dependerá de un conjunto de atributos descriptivos de este individuo que hace parte de las variables independientes y se denotan con la letra $\times_{i}$.

Luego de correr el modelo y evidenciar la validez y significancia de las variables independientes, se recurre a lo planteado por Hanemann et al. (1996) que permite estimar la disposición media a pagar y, en segundo lugar se tratará de analizar qué variables socioeconómicas afecten la probabilidad de que un individuo acepte o no el pago propuesto. Si se supone que la función de utilidad es lineal, entonces la media de la DAP coincide con la mediana y puede ser obtenida a través de la siguiente expresión:

$$
E(D A P)=-\alpha / \beta
$$

Donde $\alpha$ y $\beta$ son respectivamente los coeficientes estimados para la constante y la variable precio de entrada en el modelo logit ${ }^{2}$.

El método de costo de oportunidad. Dixon et al. (1994, p. 57) plantea que este enfoque se basa en el concepto de que el costo de utilizar recursos para otros propósitos, usualmente sin precio o fuera del mercado (por ejemplo, preservación de la tierra para un parque nacional en lugar de cosechar sus árboles para aprovechar la madera), puede aproximarse utilizando el ingreso dejado de percibir por otros los usos del recurso. Más que tratar de medir directamente los beneficios logrados por la preservación de estos recursos lo que se trata de hacer es cuantificar cuánto ingreso debe sacrificarse para satisfacer los propósitos de preservación. El enfoque de costo de oportunidad, es una manera de medir los costos de preservación.

Dixon et al. (1994), indica que el primer paso es un análisis convencional de $\mathrm{B} / \mathrm{C}$ del proyecto propuesto. $\mathrm{Si}$ el análisis tradicional del proyecto muestra que no es económi- co, el análisis no necesita continuar. Sin embargo, si el proyecto propuesto tiene beneficios netos positivos, estos deberían confrontarse con los beneficios del proyecto alternativo de preservación que pueden ser medidos fácilmente. $\mathrm{Si}$ esos beneficios mensurables del proyecto alternativo son mejores que los beneficios del proyecto propuesto, éste no se debe iniciar.

Cuando los beneficios del proyecto propuesto son apenas mayores que los del proyecto alternativo de preservación, se enfrenta en una elección difícil. La opción alternativa puede también tener beneficios menos tangibles, tales como un valor de opción, valor de cuasi-opción y valor de existencia, que son fácilmente mensurables. Esos beneficios no cuantificables deben entonces ser comparados cualitativamente con la cantidad de beneficios, en los cuales el proyecto propuesto excede a la propuesta alternativa. Cuando es poca la diferencia de beneficios entre las dos alternativas, se recomienda prudencia.

Gilpin (2007, p. 190) define el costo de oportunidad como aquel que se realiza para satisfacer un objetivo, medido por el valor que tendrían los recursos si se le diera un uso alterno interesante; por ejemplo, si los fondos de capital destinados a un programa o uso productivo podrían haber devengado una tasa de interés del 15\% anual en otra aplicación de riesgo similar.

Una forma de entender el concepto de costo de oportunidad lo presenta Bernanke (2007, p. 45). Por ejemplo, Susana dedica 4 horas al día a recoger café y 2 a recoger nueces. En cada hora recoge 4 kilos de café y 2 kilos de nueces. En este caso, terminará teniendo (4 horas al día) x (4 kilos por hora) $=16$ kilos de café al día y ( 2 horas al día) x ( 2 kilos por hora) $=4$ kilos de nueces. Supongamos ahora que Susana dedica 2 horas a recoger café y 4 horas a recoger nueces, tendrá (2 horas al día $) \times(4$ kilos por hora $)=8$ kilos de café diarios y $(4$ horas al día) $\mathrm{x}(2$ kilos por hora $)=8$ kilos de nueces diarios. A partir de estos datos decimos que el costo de oportunidad para Susana de producir nueces en términos de café es:

$$
\mathrm{CO}_{\text {nueces }}=\frac{\text { Pérdida de café }}{\text { Ganancia de nueces }}=\frac{8-16}{8-4}=\frac{-8}{4}=\frac{-2}{1}=-2
$$

Este cociente significa que el costo de oportunidad que tiene para Susana un kilo más de nueces es 2 kilos de café, es decir, que para obtener un kilo más de nueces se debe renunciar a 2 kilos de café.

De forma análoga podemos determinar con los mismos datos que el costo de oportunidad para Susana de producir café en términos de nueces:

$$
\mathrm{CO}_{\text {café }}=\frac{\text { Pérdida de nueces }}{\text { Ganancia de café }}=\frac{8-4}{8-16-4}=\frac{2}{-2}=\frac{1}{-0,5}
$$


Bioetnia Volumen 9 No 1 (enero-junio), 2012

Tabla 1

Variable dependiente

Variable dependiente

Descripción de la variable

$\mathrm{P}(\mathrm{Y}=1)$

Probabilidad de responder SI $(=1)$
Variable dicotómica, que toma el valor de 1 en caso de que la respues

ta a la disposición a pagar (DAP) por la creación y conservación del

parque es afirmativa. $Y$ toma el valor de 0 cuando la respuesta es

negativa.

Fuente: Elaboración propia (2010).

Este cociente significa que el costo de oportunidad que tiene para Susana un kilo más de nueces es 2 kilos de café, es decir, que para obtener un kilo más de café se debe renunciar a 0,5 kilos de nueces.

El método de relación $\boldsymbol{B} / \boldsymbol{C}$. La relación $\mathrm{B} / \mathrm{C}$ es un método de análisis económico utilizado para comprarar posibles decisiones alternas de inversión o acción con base en los beneficios netos que se pueden obtener. La idea se centra en medir monetariamente en el tiempo tanto los beneficios y costos de una acción o proyecto, para luego compararlos en el presente y determinar la viabilidad. Para traer los valores futuros a presente, es necesario descontar estos valores en el tiempo a una tasa de interés que se llama descuento. Esta tasa representa un costo de oportunidad de los recursos que se utilizarán en el proyecto. El valor presente neto (VPN) o valor actual neto (VAN) se calcula restando los beneficios de los costos en cada período para luego traerlos a valor presente. Su cálculo se realiza de la siguiente manera:

$$
V P N=\sum_{\mathrm{t}=0}^{n} \frac{B t-C t}{(1+r)^{t}}
$$

Reorganizando la ecuación 3 en función de la relación B/ C se tiene:

$$
R\left(\frac{B}{C}\right)=\frac{\sum_{t=0}^{n} \frac{B_{t}}{(1+r)^{t}}}{\sum_{t=0}^{n} \frac{C_{t}}{(1+r)^{t}}}
$$

Esta razón compara los beneficios descontados en el período $t\left(\mathrm{~B}_{t}\right)$ con los costos descontados en el período $t\left(\mathrm{C}_{t}\right)$. La letra $n$ representa el número de período en el tiempo y $\mathrm{r}$ la tasa de descuento. Si la relación $\mathrm{B} / \mathrm{C}$ es exactamente igual a 1, el producto producirá cero beneficios netos a lo largo de su vida. Desde la perspectiva económica, una relación $\mathrm{B} / \mathrm{C}$ mayor que 1 genera ganancias y menor que 1 pérdidas.

\section{Metodología de valoración y determinación de la muestra}

Especificación del modelo de estimación de beneficios. Para la determinación de los beneficios se empleó un modelo de regresión basados en el supuesto de distribución logística de la DAP de los individuos, comúnmente llamado modelos logit, como se ilustró en la ecuación [1]. En las Tabla 1 y 2 se relacionan las variables usadas en el modelo.

Beneficios que se perderán por la creación del parque. En esta investigación se incluyen aquellos costos de oportunidad asociados con la pérdida de la vivienda y los ingresos que dejarán de percibir por actividades comerciales, es decir, lo que estarían dispuestos a recibir los afectados para renunciar a un B/C. De 168 unidades residenciales y/o comerciales afectadas, se les preguntó su condición socioeconómica, características de la propiedad (propia o arrendada), tipo de actividad comercial e ingresos generados por mes y tiempo destinado a la actividad comercial. Esta información facilitó calcular el valor total de los beneficios que se perderán por el establecimiento del parque.

Población y muestra. La determinación de la muestra para cuantificar beneficios del proyecto se diseñó utilizando métodos de muestreo aleatorio simple (MAS), herramienta que ayuda a determinar una muestra representativa de la población donde la escogencia es aleatoria. La ecuación para determinar el tamaño de muestra es la siguiente:

$$
n_{o}=\frac{z^{2} p(1-p)}{E^{2}}
$$

Dónde:

p: Probabilidad de inclusión, es decir, la probabilidad de que una vivienda sea elegida en la muestra. La probabilidad de no inclusión es $(1-\mathrm{p})=\mathrm{q}$

E: Error máximo permitido 
Tabla 2

Variable independientes

\begin{tabular}{|c|c|c|}
\hline $\begin{array}{c}\text { Variables independientes } \\
\text { o explicativas } \\
\end{array}$ & Descripción & $\begin{array}{c}\text { Relación esperada con } \\
\text { el P (SI) } \\
\end{array}$ \\
\hline Precio $(P)$ & $\begin{array}{l}\text { Variable discreta que representa el } \\
\text { precio ofrecido al encuestado }\end{array}$ & $\begin{array}{l}\text { Negativa: a mayor precio menor } \\
\text { probabilidad de respuesta positiva }\end{array}$ \\
\hline Sexo $(S)$ & $\begin{array}{l}\text { Variable binaria que toma el valor de } 1 \\
\text { cuando es hombre y } 2 \text { cuando es } \\
\text { mujer }\end{array}$ & Desconocida \\
\hline Edad (E) & $\begin{array}{l}\text { Variables que toma los valores de } 1 \text { si } \\
\text { menor o igual a } 30 \text { años y } 2 \text { si es } \\
\text { mayor de } 30 .\end{array}$ & Desconocida \\
\hline Vinculación laboral (VL) & $\begin{array}{l}\text { Variable binaria que toma el valor de } 1 \\
\text { si es trabajador dependiente y } 2 \mathrm{si} \\
\text { trabajador independiente. }\end{array}$ & Desconocida \\
\hline Nivel de educación (NE) & $\begin{array}{l}\text { Variable que toma los valores de } 1 \text { si } \\
\text { es universitario, } 2 \text { técnico, } 3 \text { no } \\
\text { profesional y } 4 \text { analfabeta }\end{array}$ & $\begin{array}{l}\text { Positiva: a mayor nivel de educa- } \\
\text { ción mayor probabilidad de res- } \\
\text { puesta positiva }\end{array}$ \\
\hline Nivel de ingresos en la vivienda (NI) & $\begin{array}{l}\text { Variable que toma los valores de } 1 \\
\text { cuando la persona encuestada no } \\
\text { tiene ingresos propios, } 2 \text { si cuenta con } \\
\text { ingresos y aporta a la vivienda. }\end{array}$ & $\begin{array}{l}\text { Positiva: a mayor nivel de ingreso } \\
\text { se esperaría una mayor probabili- } \\
\text { dad de respuesta positiva. }\end{array}$ \\
\hline Contribuir con el parque (C) & $\begin{array}{l}\text { Variable binaria que toma el valor de } 1 \\
\text { si está de acuerdo y } 2 \text { en desacuerdo. }\end{array}$ & $\begin{array}{l}\text { Positiva: un mal estado de conser- } \\
\text { vación del parque podría aumentar } \\
\text { la probabilidad de respuesta } \\
\text { positiva. }\end{array}$ \\
\hline Responsable el Municipio (M) & $\begin{array}{l}\text { Intenta medir si la responsabilidad del } \\
\text { parque es sólo del municipio. } 1 \text { de } \\
\text { acuerdo. } 2 \text { neutral. } 3 \text { desacuerdo. } 4 \\
\text { No sabe/ no responde. }\end{array}$ & Desconocida \\
\hline
\end{tabular}

Fuente: Elaboración propia (2010).

z: Probabilidad de que la población siga una distribución normal

$\mathrm{n}$ : Tamaño de la población

$$
n_{o}=\frac{z^{2} p(1-p)}{E^{2}}=\frac{(1,96)^{2} 0.5(1-0.5)}{(0,05)^{2}}=384[6]
$$

Luego se realiza un ajuste al tamaño de muestra de la siguiente manera:

$$
n=\frac{n_{o}}{1+\frac{n_{o}}{N}}=\frac{384}{1+\frac{384}{535}}=223,60 \cong 223 \text { viviendas }^{3}
$$

Total muestra determinada para medir o cuantificar los beneficios: 223 viviendas.

Por su parte la determinación de los costos a efectos de cuantificarlos fue un censo poblacional que abarcó a todos los afectados por la creación del parque, en total 168 unidades residenciales y comerciales. El área del censo fue desde la calle 41 hasta la calle 20 aplicada a unidades que se encuentran ubicadas en la parte ribereña de la margen izquierda del río Sinú. El área de este terreno es de $95.000 \mathrm{~m}^{2}$ y 2.100 metros lineales ${ }^{4}$.

Características de la población afectada y beneficiarios directos. En la ciudad de Montería hay 61.666 viviendas ${ }^{5}$. Del total de viviendas del municipio, $63.6 \%$ corresponden a 


\section{Bioetnia Volumen 9 No 1 (enero-junio), 2012}

los estratos 1 y 2 , es decir 39.220 viviendas. El resto, 22.446, corresponden a los estratos $3,4,5$ y 6 . Sólo el estrato 3 presenta 12.518 viviendas. La ciudad la divide el río Sinú es dos partes, la margen derecha y la margen izquierda. En la margen izquierda $100 \%$ de las casas corresponden a los estratos 1 y 2 . Este proyecto analiza las características socioeconómicas de la población afectada y beneficiaria directa, sólo de la margen izquierda.

La población afectada en su mayoría son viviendas en mal estado de conservación y de invasión, el ingreso familiar depende de una sola persona (madre o padre), los ingresos mensuales no superan el salario mínimo legal vigente y es generado por actividades de empleo formal -trabajador dependiente representa 5\% del total censado- y el empleo informal -trabajos independientes representa $95 \%$ del total censado. De las actividades de trabajo independientes 50\% realizan sus actividades en la misma vivienda, el resto fuera de ella. Esta consideración es importante para valorar el costo de oportunidad de los ingresos que se perderán al ser reubicados.

Por su parte, la población beneficiaria directa del proyecto, son viviendas en estado de conservación aceptable, viviendas de estrato 1 y $2 ; 30 \%$ de los encuestados son trabajadores dependientes y el resto trabajadores independientes. Como futuros beneficiarios por el establecimiento del parque, reconocen y están dispuestos a pagar por el cambio ambiental. En el análisis de resultados se presenta los datos que expresan la DAP de la población beneficiaria directa.

Técnicas e instrumentos de recolección de datos. El instrumento para la recolección de la información, tanto de las viviendas afectadas por la reubicación como las viviendas beneficiarias por la creación del parque, es un cuestionario tipo encuesta, que captura información relevante de las características socioeconómica de la persona responsable de la vivienda tales como sexo, edad, nivel de educación, nivel de ingreso y la pregunta sobre la disposición a pagar y/o aceptar por el cambio propuesto de la creación del parque.

El cuestionario que se empleó para la medición de los beneficios y en especial para capturar la variable precio hipotético que permite calcular el valor esperado de la disposición a pagar, fue una pregunta tipo subasta, donde se le pregunta al encuestado si estaría dispuesto a pagar una suma de $\$ 10.000$ por mes y por vivienda para la creación y conservación del parque 6 . Si el encuestado responde «SÍ», entonces se incrementa el valor en $\$ 500$ hasta que responda «NO». Si por el contrario responde «NO» en la primera iteración, entonces se disminuye en $\$ 500$ hasta que responda «SÍ». La ventaja de las preguntas tipo subastas es que permite interactuar con el encuestado de forma sucesiva para encontrar el valor a pagar según sus niveles de ingreso y condición social.

\section{Análisis de los resultados}

Análisis econométrico. Para el análisis de beneficios, el modelo econométrico usado en esta investigación y el sugerido por la teoría del método de valoración contingente, es el modelo logit, que permite calcular la DAP de los encuestados a partir del cociente del término independiente con el parámetro de la variable precio. Para ello es necesario estructurar un modelo multivariable que posibilite la inclusión de categorías socioeconómicas y la variable precio hipotético. Para estimar los parámetros del modelo logit, se usó el aplicativo Eviews versión 5. El modelo multivariable que se formuló fue:

$$
D A P=\int(S, E, N E, V L, N I, C, M)
$$

Las convenciones de cada una de las variables explicativas que se relacionan en la ecuación [8] se muestran en la Tabla 2.

Luego se utilizó la estrategia parsimonioso de análisis que consiste en estimar regresiones sucesivas con un número cada vez menor de variables explicativas con el fin de lograr la mayor significancia conjunta del modelo. En la primera estimación se corren todas las variables, en la segunda se eliminan las variables explicativas menos significativas y así sucesivamente hasta que ya no sea posible eliminar ninguna más.

Después de este proceso el modelo resultante fue:

$$
D A P=\int(N E, N I, C, P)
$$

En la Tabla 3 se muestran las variables explicativas que resultaron significativas en un 95\% de confianza. Para los propósitos de esta investigación se toma el término constante «C» y se divide entre el coeficiente de la variable precio, de esta forma se obtiene el valor esperado de la disposición a pagar como se presenta a continuación.

$$
\begin{aligned}
& E(D A P)=-\alpha / \beta \\
& E(D A P)=-(-4.449549) / 0.001574 \\
& E(D A P)=\$ 2.827
\end{aligned}
$$

De esta forma se obtiene una disposición a pagar por vivienda y por mes $\$ 2.827$ pesos colombianos. Luego extrapolamos las 223 viviendas encuestados al total de viviendas del municipio con estratos socioeconómicos 1 y 2 que presentan características comunes a la muestra, que son 39.220. Este valor multiplicado por el precio de $\$ 2.827$, generará el total de beneficios (ingresos) estimados por el establecimiento del parque. Este mismo cálculo se hará para los años siguientes proyectado hasta 20 años. En la Tabla 4 se indica las bases de proyección que se utilizan. Con la 
Tabla 3

Resultados del modelo escogido

\begin{tabular}{|c|c|c|c|c|}
\hline \multicolumn{5}{|c|}{ Dependent Variable: DISPOSICION } \\
\hline \multicolumn{5}{|c|}{ Method: ML - Binary Logit (Newton-Raphson) } \\
\hline \multicolumn{5}{|c|}{ Date: 07/22/10 Time: 22:41 } \\
\hline \multicolumn{5}{|c|}{ Sample: 1223} \\
\hline \multicolumn{5}{|c|}{ Included observations: 223} \\
\hline \multicolumn{5}{|c|}{ Convergence not achieved after 4 iterations } \\
\hline \multicolumn{5}{|c|}{ GLM Robust Standard Errors \& Covariance } \\
\hline \multicolumn{5}{|c|}{ Variance factor estimate $=0.07682320996$} \\
\hline \multicolumn{5}{|c|}{ Covariance matrix computed using second derivatives } \\
\hline Variable & Coefficient & Std. Error & z-Statistic & Prob. \\
\hline C & -4.449549 & 0.355474 & -12.51722 & 0.0000 \\
\hline Estudio (NE) & 0.294967 & 0.075681 & 3.897509 & 0.0001 \\
\hline Ingreso (NI) & -0.455497 & 0.187607 & -2.427929 & 0.0152 \\
\hline Contribución (C) & 1.172925 & 0.213050 & 5.505406 & 0.0000 \\
\hline Precio $(P)$ & 0.001574 & 4.10E-05 & 38.36446 & 0.0000 \\
\hline Mean dependent var & 0.708520 & S.D. dependent var & 0.455466 & \\
\hline SE of regression & 0.108008 & Akaike info criterion & 0.165188 & \\
\hline Sum squared resid & 2.543124 & Schwarz criterion & 0.241582 & \\
\hline Log likelihood & -13.41848 & Hannan-Quinn criter. & 0.196028 & \\
\hline Restr. log likelihood & -134.5741 & Avg. log likelihood & -0.060173 & \\
\hline LR statistic (4 df) & 242.3113 & McFadden R-squared & 0.900289 & \\
\hline Probability(LR stat) & 0.000000 & & & \\
\hline Obs with Dep $=0$ & 65 & Total obs & 223 & \\
\hline Obs with Dep=1 & 158 & & & \\
\hline
\end{tabular}

Fuente: Elaboración propia (2010).

información de la Tabla 4, se proyecta a 20 años los beneficios y costos de la creación del parque, los cuales se indican en la Tabla 5.

El ingreso DAP de las viviendas, se proyectó hasta el año 2031, se tomó como año base el año 2011 como año 0 , y de ahí en adelante se proyectó con una tasa de factor de ajuste del $5 \%$. Por su parte los costos se proyectaron hasta el año 2031 sólo los costos de la fase de post-inversión que se iniciaron en el año 2012 (año 1).

Los beneficios netos, es decir la diferencia entre los ingresos y los costos, se traen a valor presente a una tasa de descuento del $6 \%$ efectivo anual. La justificación de usar esta tasa para descontar los beneficios netos, se explica por el criterio de depositar dinero en el sistema financiero. En la literatura reciente es común utilizar el método del costo de oportunidad del capital y la tasa social de preferencia en el tiempo ${ }^{7}$. Según estadísticas del Banco de la República de Colombia, la tasa de interés de los certificados de depósito a término promedio a 360 días para junio de 2009 fue de $6 \%$ efectiva anual, lo cual sirve como tasa de descuento de referencia para calcular el valor presente neto en esta inves- tigación. De acuerdo con lo anterior, se calculó y se realizó la evaluación económica de los beneficios y costos de establecer un parque en la margen izquierda (Tabla 6).

$\mathrm{Si}$ bien es cierto, se trata de un proyecto que beneficia a la sociedad en general, por su impacto ambiental y social; la relación $\mathrm{B} / \mathrm{C}$ de 1,47 indica que el proyecto es viable al arrojar unos ingresos descontados de \$24,014’720.656 y unos costos descontados de $\$ 16,332 ’ 489.249$. Hay que recordar que estos resultados son sólo con las viviendas de estrato 1 y 2. No obstante, si presentamos el análisis con todas las viviendas del municipio, incluyendo los estratos 3, 4, 5 y 6 , los resultados son mucho más significativos y el proyecto se vuelve viable económicamente por los tres métodos de evaluación que se han considerado.

En la Tabla 7, se muestran los resultados con los estratos 1 a 6 , es decir, para el cálculo del beneficio se utilizan 61.666 viviendas y no 39.220 como se realizó en la Tabla 6. La relación $\mathrm{B} / \mathrm{C}$ arrojó un valor de 2,31 lo cual indica que el proyecto es viable al presentar ingresos descontados de $\$ 37,758,586.537$ y unos costos descontados de $\$ 16,332$ '489.249. De igual forma se presenta un valor actual 
Tabla 4

Bases para proyectar los beneficios y costos del proyecto

\begin{tabular}{lc}
\hline \multicolumn{1}{c}{ Beneficios (ingresos) } & Valor \\
\hline Precio hipotético de entrada & $\$ 2.287$ \\
Número de propiedades en estrato 1 y 2 & 39.220 \\
Ingresos por mes de viviendas & $\$ 110.874 .940$ \\
Ingresos en un año de viviendas & $\$ 1^{\prime} 330.499 .280$ \\
& \\
& Valor \\
Costo de oportunidad por propiedad & $\$ 27^{\prime} 000.000$ \\
Total censo propiedades afectadas por reubicación & 168 \\
Total costo de oportunidad propiedades & $\$ 4^{\prime} 536.000 .000$ \\
Costo de oportunidad por negocios en un año & $\$ 4^{\prime} 500.000$ \\
Total censo negocios afectados por reubicación & 69 \\
Total costo de oportunidad negocios afectados & $\$ 310^{\prime} 500.000$ \\
Costo construcción del parque por m ${ }^{2}$ & $\$ 85.000$ \\
Área total en $m^{2}$ para establecer el parque & 95.000 \\
Total costo construcción del parque & $\$ 8^{\prime} 075.000 .000$ \\
\hline Total costos para establecer el parque & $\$ 12^{\prime} 921.500 .000$ \\
Imprevistos 10\% & $\$ 1^{\prime 2} 292.150 .000$ \\
\hline Total costos creación fase inversión inicial & $\$ 14^{\prime} 213.650 .000$ \\
\hline Total costos fase post-inversión (en marcha) & $\$ 150^{\prime} 000.000$ \\
\hline
\end{tabular}

Fuente: Elaboración propia (2010).

Tabla 5

Beneficios y costos del proyecto «Establecer un parque en la margen izquierda»

\begin{tabular}{lcccc}
\hline Actividades & $\mathbf{2 0 1 1}$ & $\mathbf{2 0 1 2}$ & $\mathbf{2 0 1 3}$ & $\mathbf{2 0 3 1}$ \\
\hline $\begin{array}{l}\text { Ingresos por creación del parque } \\
\text { DAP de los viviendas }\end{array}$ & $\$ 1.330^{\prime} 499.280$ & $\$ 1.397^{\prime} 024.244$ & $\$ 1.466^{\prime} 875.456$ & $\$ 3.530^{\prime} 210.686$ \\
Costos por creación del parque & & & & \\
Total costo creación fase inversión inicial & $\$ 14.213^{\prime} 560.000$ & & $\$ 160^{\prime} 500.000$ & $\$ 542^{\prime} 479.130$ \\
Costos fase post - inversión & & $\$ 150^{\prime} 000.000$ & $\$ 2.987^{\prime} 731.556$ \\
Beneficios netos & $\$\left(12.883^{\prime} 150.720\right)$ & $\$ 1.247^{\prime} 024.244$ & $\$ 1.306^{\prime} 375.456$ & $\$ 931^{\prime} 588.822$ \\
Beneficios netos descontados & $\$\left(12.883^{\prime} 150.720\right)$ & $\$ 1.176 .^{\prime} 437.966$ & $\$ 1.162^{\prime} 669.505$ & $\$$ \\
\hline
\end{tabular}

Fuente: Elaboración propia (2010).

o presente neto de $\$ 7,809$ ' 889.743 y una TIR del $14 \%$, que ratifica la pertinencia y viabilidad del proyecto parque margen izquierda.

\section{Conclusiones}

En cuanto a los resultados del modelo econométrico escogido (Tabla 3), todas las variables explicativas resultaron significativas con un $95 \%$ de confianza. La variable ingreso aunque resultó con signo negativo -no era el esperado-, es consistente en el sentido que la muestra encuestada son de estrato 1 y 2 , que presentan características socioeconómicas de bajos ingresos y la actividad laboral es trabajo independiente. De tal forma que si el nivel de ingreso 


\section{Tabla 6 Resultados de la evaluación económica sólo con estratos 1 y 2}

\begin{tabular}{lr}
\hline VAN & $\$(513.118 .104)$ \\
TIR & $5 \%$ \\
B/C & 1,47 \\
\hline
\end{tabular}

Fuente: Elaboración propia (2010).

\section{Tabla 7 \\ Resultados de la evaluación económica con todos los estratos socioeconómicos}

\begin{tabular}{lr}
\hline VAN & $\$ 7.809 .889 .743$ \\
TIR & $14 \%$ \\
B/C & 2,31 \\
\hline
\end{tabular}

Fuente: Elaboración propia (2010).

aumenta en una unidad monetaria, la probabilidad de una repuesta afirmativa disminuye en 0,455497 . Pero si lo comparamos con el coeficiente de contribuir con el parque, se encuentra una lógica consistente con el nivel de ingresos, porque un cambio en la contribución para crear y sostener el parque en una unidad, aumenta la probabilidad de una respuesta afirmativa en 1.172925. Esto quiere decir, que las viviendas de los estratos 1 y 2 estarían altamente dispuestas a contribuir con el parque en cualquier forma distinta a pagar en dinero.

Relacionado con el punto anterior, el cálculo del valor esperado de la disposición a pagar es de $\$ 2.827$ por mes y por vivienda; si bien es cierto es una aporte bajo, también es cierto que se ajusta a la capacidad económica de los viviendas encuestadas.

Luego de haber estimados los beneficios y costos, por los métodos de valoración contingente y costos de oportunidad, y evaluado económicamente el método de valor actual neto (VAN), tasa interna de retorno (TIR) y relación $\mathrm{B} / \mathrm{C}$, se puede concluir que la creación o establecimiento de un parque en la margen izquierda del río Sinú es viable desde la perspectiva económica.

Descontando los beneficios y costos a una tasa de descuento del $6 \%$, el proyecto es viable con una relación $\mathrm{B} / \mathrm{C}$ de 1,47 cuando sólo se incluyen en el estudio las viviendas de estratos 1 y 2. Si incluimos todas las viviendas de estratos socioeconómicas, la relación $\mathrm{B} / \mathrm{C}$ es de 2,31. Para este caso, el retorno de la inversión del $14 \%$ es muy buena teniendo en cuenta que en los ingresos sólo se valora la DAP por establecer el parque y no se valora otros ingresos que son importan- tes y afectan de forma significativa la evaluación del proyecto como la reducción de terreno erosionado, mejoramiento en conductas sociales para el cuidado del medio ambiente, conservación de biodiversidad, ingresos por actividades económicas diversas -turismo, comercio-, entre otras. Estas categorías de ingresos no se incluyeron en este proyecto, pero se recomienda estudiarlas en investigaciones futuras con el fin de tener una evaluación más cercana a la realidad.

\section{Literatura citada}

Alier, J. J. Roca. 2001. Economía ecológica y política ambiental. $2^{\mathrm{a}}$ ed., México, DF: Editorial Fondo de Cultura Económica. 499 pp.

Azqueta, D. 1994. Valoración económica de la calidad ambiental. Madrid: McGraw Hill.

Azqueta, D., L. Pérez-Pérez. 1996. Gestión de espacios naturales. La demanda de servicios recreativos. Madrid: McGraw-Hill. 237 pp.

Bernanke, B., R. Frank. 2007. Microeconomía. $3^{\mathrm{a}}$ ed. Madrid: Editorial McGraw Hill. 409 pp.

Camarero, L. 2006. Medio ambiente y sociedad. Madrid: Editorial Thomson. 384 pp.

Cochran, W. 1985. Técnicas de muestreo. México, DF: Cecsa., 513 pp.

Dixon, J., S. Fallon, R. Carpenter, P. Sherman. 1994. Análisis económico de impactos ambientales. Turrialba: Centro Agronómico Tropical de Investigación y Enseñanza (CATIE). 249 pp.

Field, B. C., M. K. Field. 2003. Economía ambiental. Una introducción. Bogotá: McGraw Hill.

García, L., A. Colina. 2004. Métodos directos e indirectos en la valoración económica de bienes ambientales. Aplicación al valor de uso recreativo del parque natural de Somiedo. Estudios de Economía Aplicada, 3 (22): 729-30.

Gilpin, A. 2007. Economía ambiental. México, D. F.: Editorial Alfaomega. $326 \mathrm{pp}$.

Gujarati, D. 1992. Econometría. 2a ed. México: McGraw Hill.

Haneman, W. M., B. Kanninen. 1996. The statistical analysis of discrete responde CV data. Working Paper $\mathrm{N}^{\circ}$ 798. Chicago: Departament of Agricultural and Resource Economics, University of California. 123 pp.

Martínez, C. 2000. Estadística y muestreo. 10a ed. Bogotá: Eco Ediciones. p. 85-110.

Mathews, K., F. R. Johnson, R. W. Dunford, W. H. Desvousges. 1995. The potential role of conjoint analysis in natural resource damage assessments. Triangle Economic Research (TER). General Working Paper N G-9503. Durham: Triangle Economic Research.

Riera, P. 1994. Manual de valoración contingente. Madrid: Editorial Institutos de Estudios Fiscales. p. 103-67.

Riera, P., D. García, B. Kristron, R. Brannlund. 2005. Manual de economía ambiental y de los recursos naturales. Madrid: Editorial Thomson. 355 pp.

Sepúlveda-Vargas, R. D. 2008. Valoración económica del uso recreativo del parque ronda del Sinú, en Montería, Colombia. Semestre Económico. 11 (22): $67-90$.

\section{Notas de pie de página}

2 Si el lector desea profundizar más sobre estos tipos de formato y la forma de estructurarlo, puede consultar Azqueta, Diego (1994).

3 El Departamento Administrativo Nacional de Estadística (DANE), explica la diferencia entre una vivienda y hogar. Vivienda es un lugar estructuralmente separado e independiente, ocupado o destinado a ser ocupado por una familia o grupo de personas que viven juntas, o por una persona que vive sola. 


\section{Bioetnia Volumen 9 No 1 (enero-junio), 2012}

La unidad de vivienda puede ser una casa, apartamento, cuarto, grupo de cuartos, choza, cueva, o cualquier refugio ocupado o disponible para ser utilizado como lugar de alojamiento. Por su parte un hogar, es una persona o grupo de personas que ocupan la totalidad o parte de una vivienda y que se han asociado para compartir la comida y la dormida. Pueden ser familiares o no entre si. Los empleados del servicio doméstico y sus familiares forman parte del hogar siempre y cuando duerman en la misma vivienda. En la vivienda se pueden encontrar inquilinos o huéspedes: el inquilino es la persona que tiene en arriendo uno o varios cuartos de la vivienda y hace sus comidas por aparte, razón por la cual se debe considerar como hogar independiente. Los llamados huéspedes y pensionistas hacen parte del hogar.
4 Información suministrada por la Secretaría de Hacienda Municipio de Montería.

5 Información suministrada por la Secretaría de Planeación del municipio de Montería, datos año 2002.

6 El precio hipotético de $\$ 10.000$ por mes y por vivienda, es un precio de partida que se usa como referencia cuando se utilizan preguntas de tipo subasta. Este precio refleja el precio de entrada a un parque privado en la localidad, lo cual permite simular un mercado hipotético para un parque público.

7 Si el lector desea ampliar más el concepto de la tasa de descuento, puede consultar el libro de «Análisis económico de impactos ambientales» de John Dixon y otros (también se referencia en la bibliografía de esta investigación). 